\title{
Time to embrace neoadjuvant chemotherapy for invasive disease
}

The survival benefit of administering cisplatin-based chemotherapy before focal treatment of muscle-invasive bladder cancer has been confirmed by long-term results of the international multicenter BA06 30894 trial. Experts are heralding a paradigm shift, with neoadjuvant chemotherapy as the standard of care for advanced disease. This will be welcome news for affected patients, given the currently high death rates associated with local therapy alone.

\section{4 ...CMV was associated with} a statistically significant $16 \%$ reduction in the risk of death... 77

The open, randomized, phase III trial compared outcomes of patients who underwent definitive local treatment (either radiotherapy or cystectomy) with or without three cycles of neoadjuvant cisplatin, methotrexate and vinblastine (CMV) chemotherapy. Interim results of the trial-which accrued 976 patients between 1989 and 1995-were reported in 1999. At that time, only a modest nonsignificant improvement in survival was shown for patients who received CMV. Gareth Griffiths and colleagues now report the long-awaited mature data in the Journal of Clinical Oncology.

At a median follow up of 8 years, neoadjuvant CMV was associated with a statistically significant $16 \%$ reduction in the risk of death (HR 0.84; 95\% CI $0.72-0.99 ; P=0.037$ ), which corresponded to a $6 \%$ increase in 10-year survival (from $30 \%$ to $36 \%$ ) and improvement of median survival time by 7 months (from 37 to 44 months). There was no evidence to suggest that neoadjuvant CMV was more or less effective when combined with either radiotherapy or cystectomy alone.

The results are similar to those of the Southwest Oncology Group trial of methotrexate, vinblastine, doxorubicin and cisplatin, which showed a $25 \%$ reduction in the risk of death, thus adding to a compelling body of evidence that supports the use of neoadjuvant chemotherapy in this setting. In an accompanying editorial, Dean Bajorin and Harry Herr urge the urologic community to routinely offer neoadjuvant CMV and encourage collaboration between urologists and medical oncologists.

Despite the considerable buzz surrounding these new data, the authors remain pragmatic, pointing out that over $70 \%$ of participating clinicians agreed that only a $10 \%$ improvement in survival would justify the routine adoption of neoadjuvant CMV. "The results of this trial and others indicate that only modest improvements in the neoadjuvant setting

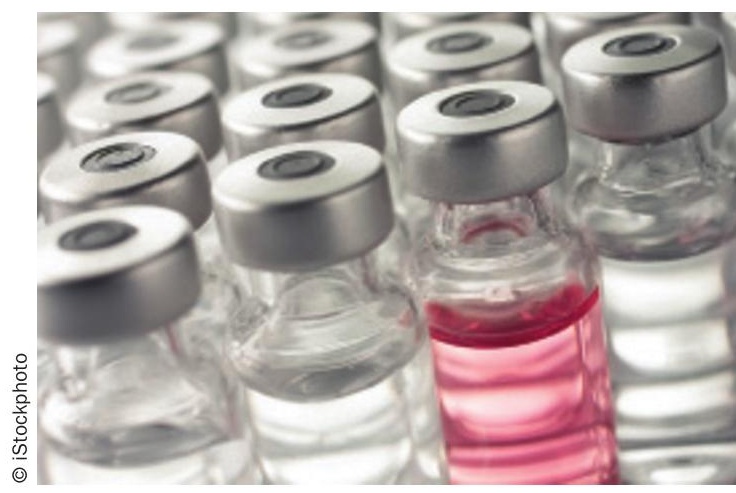

are likely to be achieved. We believe larger improvements require the use of targeted agents in individualized treatment strategies within specific patient subpopulations, and that future research should also focus on new strategies, such as the development of novel surrogate end points and biomarkers," says Griffiths.

Sarah Payton

Original article International Collaboration of Trialists on behalf of the Medical Research Council Advanced Bladder Cancer Working Party. International phase III trial assessing neoadjuvant cisplatin, methotrexate, and vinblastine chemotherapy for muscle-invasive bladder cancer: longterm results of the BA06 30894 trial. J. Clin. Oncol. 29, 2171-2177 (2011) 